\title{
Interaction of iodide with green rust and magnetite
}

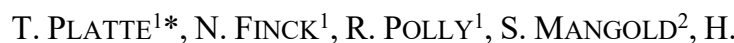 \\ GECKEIS $^{1}$
}

${ }^{1}$ Karlsruhe Institute of Technology (KIT), Institute for

Nuclear Waste Disposal (INE), P.O. 3640, D-76021

Karlsruhe (*correspondence: tim.platte@kit.edu).

${ }^{2}$ Karlsruhe Institute of Technology (KIT), Institute for Photon Science and Synchrotron Radiation (IPS), P.O. 3640, D-

76021 Karlsruhe, Germany

The long-lived fission product ${ }^{129} \mathrm{I}$ is a dose determining nuclide in many safety analyses for nuclear waste repositories [1]. It is expected to prevail as anionic species in such repositories and thus be poorly retained by geological and engineered barriers such as bentonite. In contrast, layered double hydroxides (LDH) have high affinity for anions. Green rust (GR) is a mixed-valent iron-bearing LDH and can form as corrosion product of steel canisters upon contact with groundwater. The interaction of iodide with GR was investigated in this study.

Chloride GR (GR-Cl) was prepared in the presence of iodide at constant ionic strength. Analyses by XRD and XAS indicate the formation of a solid solution between the chloride and iodide endmembers. Separately, iodide was contacted with pre-formed GR-Cl. In these experiments, sorption equilibrium is reached fast, the uptake decreases with increasing ionic strength. Experimental data suggest iodide interaction with GR-Cl via ion exchange.

Iodide containing GR samples were transformed into magnetite, the most thermodynamically stable Fe phase under repository conditions. First data show only weak incorporation of iodide into magnetite after GR transformation.

Acknowledgement: This work is part of a PhD thesis and supported by the VESPA-2 project, funded by the German Federal Ministry for Economic Affairs and Energy.

[1] Hou, X., et al. Anal. Chim. Acta 632, 181 (2009). 